\begin{tabular}{|c|c|}
\hline Title & A compact fluorescence polarization analyzer with high-transmittance liquid crystal Iayer \\
\hline Author(s) & $\begin{array}{l}\text { Wakao, O samu; Satou, Ken; Nakamura, Ayano; Sumiyoshi, Ken; Shirokawa, Masanori; Mizokuchi, Chikaaki; Shiota, } \\
\text { Kunihiro; Maeki, Masatoshi; Ishida, A kihiko; Tani, Hirofumi; Shigemura, Koji; Hibara, A kihide; Tokeshi, Manabu }\end{array}$ \\
\hline Citation & $\begin{array}{l}\text { Review of Scientific Instruments, 89(2), } 024103 \\
\text { https://doi.org/10.1063/1.5017081 }\end{array}$ \\
\hline Issue Date & $2018-02-27$ \\
\hline Doc URL & http://hdl.handle.net/2115/72720 \\
\hline Rights & $\begin{array}{l}\text { This article may be downloaded for personal use only. A ny other use requires prior permission of the author and AIP } \\
\text { Publishing. The following article appeared in Review of Scientific Instruments 89, } 024103(2018) \text { and may be found at } \\
\text { https://doi.org/10.1063/1.5017081. }\end{array}$ \\
\hline Type & article \\
\hline File Information & 1.5017081.pdf \\
\hline
\end{tabular}

Instructions for use 


\section{A compact fluorescence polarization analyzer with high-transmittance liquid crystal layer}

Osamu Wakao, Ken Satou, Ayano Nakamura, Ken Sumiyoshi, Masanori Shirokawa, Chikaaki Mizokuchi, Kunihiro Shiota, Masatoshi Maeki, Akihiko Ishida, Hirofumi Tani, Koji Shigemura, Akihide Hibara, and Manabu Tokeshi

Citation: Review of Scientific Instruments 89, 024103 (2018); doi: 10.1063/1.5017081

View online: https://doi.org/10.1063/1.5017081

View Table of Contents: http://aip.scitation.org/toc/rsi/89/2

Published by the American Institute of Physics

\section{Articles you may be interested in}

A remotely triggered fast neutron detection instrument based on a plastic organic scintillator

Review of Scientific Instruments 89, 023115 (2018); 10.1063/1.5012121

Time-resolved optical absorption microspectroscopy of magnetic field sensitive flavin photochemistry

Review of Scientific Instruments 89, 023707 (2018); 10.1063/1.5011693

A robust cloud registration method based on redundant data reduction using backpropagation neural network and shift window

Review of Scientific Instruments 89, 024704 (2018); 10.1063/1.4996628

Quantitative magneto-optical investigation of superconductor/ferromagnet hybrid structures

Review of Scientific Instruments 89, 023705 (2018); 10.1063/1.5016293

Quantitative characterization of semiconductor structures with a scanning microwave microscope Review of Scientific Instruments 89, 023706 (2018); 10.1063/1.5013113

Ion temperature measurements of indirect-drive implosions with the neutron time-of-flight detector on SG-III laser facility

Review of Scientific Instruments 89, 023504 (2018); 10.1063/1.5022767

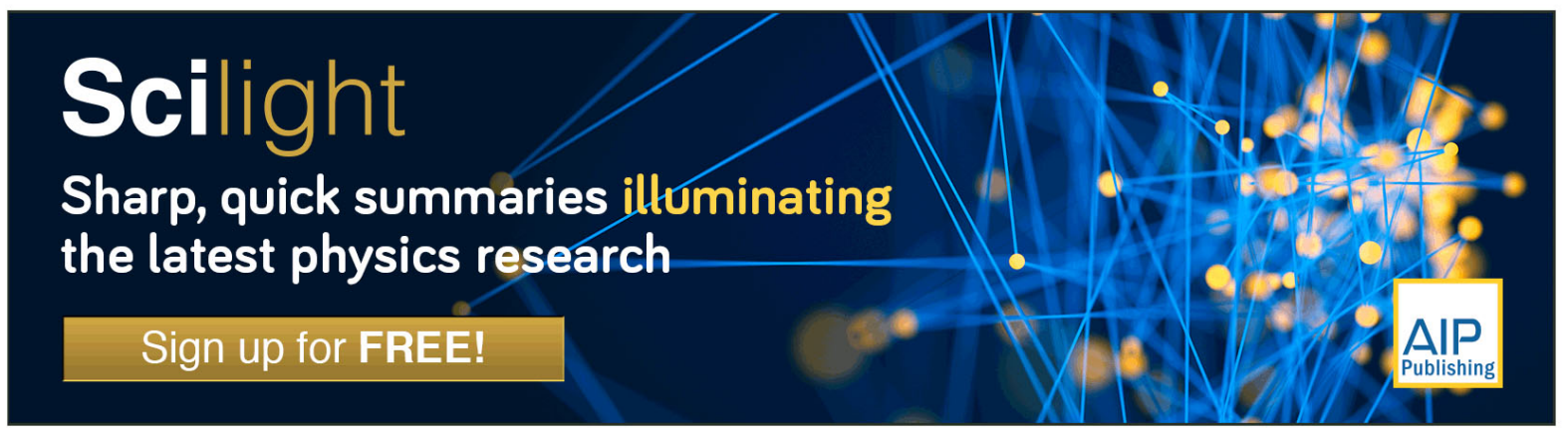




\title{
A compact fluorescence polarization analyzer with high-transmittance liquid crystal layer
}

\author{
Osamu Wakao, ${ }^{1}$ Ken Satou, ${ }^{2}$ Ayano Nakamura, ${ }^{3}$ Ken Sumiyoshi, ${ }^{2}$ Masanori Shirokawa, ${ }^{2}$ \\ Chikaaki Mizokuchi, ${ }^{2}$ Kunihiro Shiota, ${ }^{2}$ Masatoshi Maeki, ${ }^{4}$ Akihiko Ishida, ${ }^{4}$ Hirofumi Tani, ${ }^{4}$ \\ Koji Shigemura, ${ }^{2}$ Akihide Hibara, ${ }^{5, a)}$ and Manabu Tokeshi, a) \\ ${ }^{1}$ Graduate School of Chemical Sciences and Engineering, Hokkaido University, Kita 13 Nishi 8, Kita-ku, \\ Sapporo 060-8628, Japan \\ ${ }^{2}$ Tianma Japan, Ltd., Shin-Kawasaki Mitsui Building West Tower 28 F 1-1-2, Kashimada, Saiwai-ku, Kawasaki, \\ Kanagawa 212-0058, Japan \\ ${ }^{3}$ Department of Applied Science and Engineering, School of Engineering, Hokkaido University, Kita 13 Nishi 8 , \\ Kita-ku, Sapporo 060-8628, Japan \\ ${ }^{4}$ Faculty of Engineering, Division of Applied Chemistry, Hokkaido University, Kita 13 Nishi 8, Kita-ku, \\ Sapporo 060-8628, Japan \\ ${ }^{5}$ Institute of Multidisciplinary Research for Advanced Materials, Tohoku University, Katahira 2-1-1, Aoba-ku, \\ Sendai 980-8577, Japan
}

(Received 24 November 2017; accepted 3 February 2018; published online 27 February 2018)

\begin{abstract}
Fluorescence polarization (FP) offers easy operation and rapid processing, making it implementable in molecular interaction analysis. Previously we have developed a unique FP measurement system using a liquid crystal (LC) layer and an image sensor. The system is based on a principle of synchronized detection between the switching rate of the LC layer and the sampling rate of the CCD. The FP system realized simultaneous multiple sample detection; however, the measurement precision was lower than that of the conventional FP apparatus. The main drawbacks were low light transmittance of the LC layer and insufficient synchronization between the LC layer and CCD. In this paper, we developed a new FP analyzer based on LC-CCD synchronization detection. By using a newly designed LC with high transmittance and improving synchronization, the performance of the system has been dramatically improved. Additionally, we reduced the cost by using an inexpensive CCD and an LED as the excitation source. Simultaneous FP immunoassay of multiple samples of prostaglandin E2 was performed. The error rate of the FP system is reduced from $16.9 \%$ to $3.9 \%$, as comparable to the commercial conventional FP system. Published by AIP Publishing. https://doi.org/10.1063/1.5017081
\end{abstract}

\section{INTRODUCTION}

Understanding systematically biological systems and phenomena is important to obtain fundamental knowledge in biology and also in drug discovery applications. ${ }^{1,2}$ Biophysics attempts to elucidate biological systems and functions using physicochemical, reactive mechanics, and statistical approaches. Molecular interactions are pivotal in understanding the functions of biological systems and phenomena. Analyzing specific molecular interactions is one of the major challenges in biophysics. Fluorescence imaging, ${ }^{3}$ transmission electron microscopy, ${ }^{4}$ and fluorescence resonance energy transfer $(\text { FRET })^{5}$ are typically employed to analyze molecular interactions such as protein-protein interaction, protein-ligand interaction, and protein-DNA interaction. However these techniques require complex and expensive equipment as well as several time-consuming technical processes for sensitive molecular interaction analysis. Therefore simple, rapid, and high-throughput technique for molecular interaction analysis is required. Fluorescence polarization (FP) is a solution-based method which is widely used to study molecular interactions. ${ }^{6-12}$ In FP measurements, $P$, degree of polarization, is

\footnotetext{
a) Authors to whom correspondence should be addressed: hibara@tohoku.ac.jp and tokeshi@eng.hokudai.ac.jp
}

determined by the following equation:

$$
P=\left(I_{\|}-I_{\perp}\right) /\left(I_{\|}+I_{\perp}\right),
$$

where $I_{\|}$and $I_{\perp}$ are the fluorescence intensities parallel and perpendicular to the excitation polarization, respectively. Therefore, it is necessary to select each polarization to determine $P$. Conventional FP measurement apparatus employ a rotating polarizer ${ }^{6-10}$ or a polarizing splitter ${ }^{11,12}$ to select the polarizations and detect their intensities with a photodiode $^{6-8,11}$ or an image sensor. ${ }^{9,10,12}$ FP measurements with this mechanism is limited to single sample analysis and low throughput. Although commercial fluorescence spectrometers present microplate-based FP measurements to improve the throughput, sample scanning is necessary that requires additional complex optical components. ${ }^{13,14}$

Previously we had developed a unique FP measurement system with a liquid crystal (LC) layer and a CCD image sensor which enables simultaneous FP imaging for multiple samples. ${ }^{15}$ The FP measurement system is based on the synchronized detection between the switching rate of the LC layer and the sampling rate of the CCD. This system consists of a semiconductor laser, a CCD camera, a LC layer, and optical components such as an objective lens, filters, and mirror. Using this system, we succeeded in demonstrating simultaneous detection of molecular interactions between an antigen and 
antibody in multiple samples using FP immunoassay (FPIA). However, the measurement precision of our system was lower than that of the conventional FP apparatus. The main reason for a low precision was that only a fraction of the incident light was transmitted by the LC layer, obtained by disassembling a commercially available LCD. Moreover, the control system for synchronization between the switching rate of the LC layer and the sampling rate of the CCD needed to be improved.

In this paper, we developed an improved system addressing the problems of the previous system. By using a LC suitable for our purpose, the performance of the system has been significantly improved. We succeeded in cost reduction by using an inexpensive CCD and an LED instead of an electronically cooled CCD and a laser, respectively. In order to evaluate the performance of the developed system, simultaneous FPIA of multiple samples of prostaglandin E2 was performed and compared with a conventional FP apparatus.

\section{PRINCIPLE}

The detection principle is based on LC-CCD synchronization detection as mentioned in Ref. 15. In a FP imaging system, the FP signal passing through the LC layer is modulated at a certain frequency $(f \mathrm{~Hz})$ by on-off switching of the voltage applied to the LC layer. When such a fluorescence signal is captured by the CCD which is operated at $4 f \mathrm{~Hz}$ to synchronize with the LC switching, four images with FP signals are sampled. By processing these four images, the system obtains an amplitude (AC) image which includes different components of FP and an offset (DC) image which includes sum components of FP. Thus, $P$ values of multiple samples are obtained as single two-dimensional image by image analysis.

\section{THE INSTRUMENT}

We have built an FP imaging system using an LED allowing multiple sample processing. The setup is shown in Fig. 1. A $470 \mathrm{~nm}$ wavelength LED (M470L3, Thorlabs, Newton, NJ) was employed as the excitation source. In order to collimate

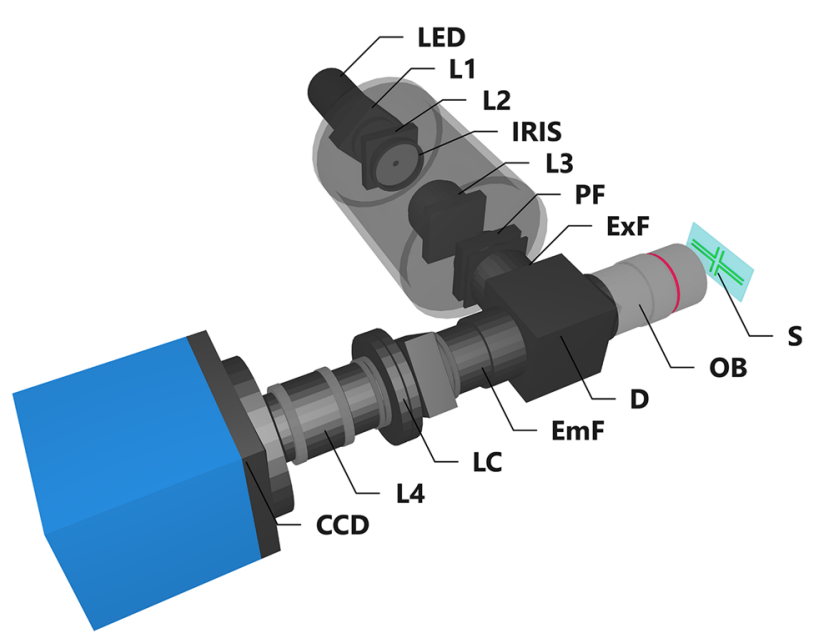

FIG. 1. Schematic illustration of the experimental setup for FP imaging (where L: lens, PF: polarizing filter, ExF: excitation filter, D: dichroic mirror, OB: objective lens, S: sample and EmF: emission filter, respectively). the beam, an aspheric condenser lens (ACL5040U-A, Thorlabs) was used. The excitation beam was introduced into plano convex lenses (SLB-25.4-35P, Sigma Koki, Tokyo, Japan) and focused on the blades of an iris diaphragm (SM1D12, Thorlabs). The iris diaphragm removed the unnecessary beam to create a uniform beam profile. After passing through the iris diaphragm, the beam was collimated again by the achromatic lens (ATL-30-40PY2, Sigma Koki). The collimated beam was introduced into a linear glass polarizing filter (Edmund Optics, Barrington, NJ). The polarized excitation beam enters a dichroic block (A11214, Hamamatsu Photonics, Hamamatsu, Japan) with an excitation filter (ET470/10x, Chroma technology, Bellows Falls, VT) and a dichroic mirror (DM505, Olympus, Tokyo, Japan). The dichroic mirror reflects the excitation beam and the beam passes through the objective lens (M Plan Apo 5×, Mitutoyo, Kawasaki, Japan). The excitation beam was focused onto a microdevice that holds the samples to be imaged.

The microdevice was fabricated by standard softlithography techniques with PDMS and a glass slide. ${ }^{16}$ The microdevice had microchannels with individual inlets and outlets to hold four different samples. Each microchannel is $300 \mu \mathrm{m}$ wide and $1 \mathrm{~mm}$ deep with $<1 \mu \mathrm{l}$ sample volume. The sample has an emission wavelength of $\sim 520 \mathrm{~nm}$. The fluorescence signals were collected by the objective lens and then introduced into the dichroic block including the emission filter (ET520/10x, Chroma technology). After passing through the dichroic mirror and the emission filter, the fluorescence passes through a LC layer (MS- $\beta 14$ A, Tianma Japan, Kawasaki, Japan) with a polarization filter while polarization modulating. The LC layer is designed as a single pixel to increase the transmittance. The transmittance increased from $\sim 7 \%$ to $\sim 40 \%$. This addressed one of the main drawbacks of the previous FP system. The fluorescence signals were focused on the CCD image sensor (Retiga R1, QImaging, Surrey, BC, Canada) by an achromatic lens (ATL-3040PY2, Sigma Koki) and captured as images. The captured images were processed by our homebuilt software designed using the Microsoft visual studio. Frequency of the LC panel orientation and that of image sampling for CCD were synchronized by a function generator (WF1948, NF, Yokohama, Japan) with the ratio of 1: 4 . The applied voltage to the LC panel was designed to fit our measurement conditions $(60 \mathrm{~Hz},<8 \mathrm{~V})$. FP measurement on a similar sample was also performed by a commercial conventional FP apparatus (FP-715, JASCO Co., Tokyo, Japan) to compare their performance.

\section{RESULTS AND DISCUSSION}

FP immunoassay (FPIA) is a popular fluorescence polarization measurement technique ${ }^{17,18}$ for detection of molecular interactions. FPIA is based on competitive binding reaction of an unlabeled target molecule and a fluorescently labeled target molecule (tracer) to an antibody. The mixture of tracer and antibody molecules results in high FP values due to slow molecular motions. The presence of unlabeled target molecules in the mixture leads to the competition with the tracer molecules for the binding site of the antibody. The competition leads 
(a)

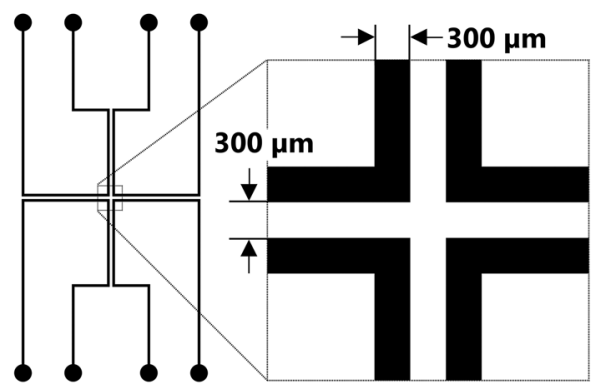

(c)

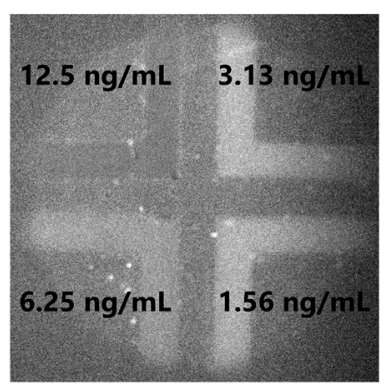

(b)

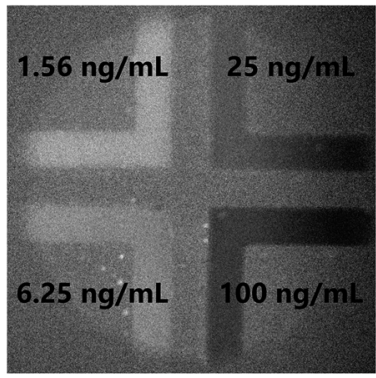

(d)

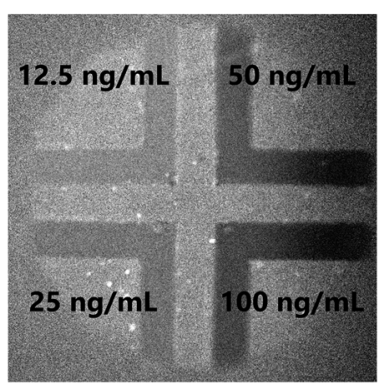

FIG. 2. Images obtained by the developed FP imaging system. (a) Design and dimensions of microdevice. FP imaging of four samples with several PGE2 concentrations (b) $(1.56,6.25,25$, and $100 \mathrm{ng} / \mathrm{ml})$, (c) $(1.56$, $3.13,6.25$, and $12.5 \mathrm{ng} / \mathrm{ml})$, and (d) $(12.5,25,50$, and $100 \mathrm{ng} / \mathrm{ml})$. to reduced FP with increasing target concentration. Based on these binding interaction mechanisms, FPIA is established in food testing and clinical applications.

In this paper, we demonstrated simultaneous multiple sample FPIA for prostaglandin E2 (PGE2) as a model target to evaluate the performance of the developed system. PGE2, lipid metabolite of arachidonic acid, is an endogenous mediator and modulator of innate immunity, inflammatory effects ${ }^{19}$ as well as in sleeping and waking regulation. ${ }^{20}$ The mixture of PGE2, fluorescein conjugated PGE2, and anti-PGE2 antibody was incubated before sample imaging. The reactions were performed according to the instructions of the commercial PGE2 assay kit manufacturer (Abnova Prostaglandin E2 FPIA Kit, Abnova, Taipei, Taiwan). The microdevice was designed to obtain four samples with different target concentrations in the same field of view. We demonstrated simultaneous FP measurements of 7 different PGE2 concentrations $(1.56,3.13,6.25,12.5,25,50$, and $100 \mathrm{ng} / \mathrm{ml})$,

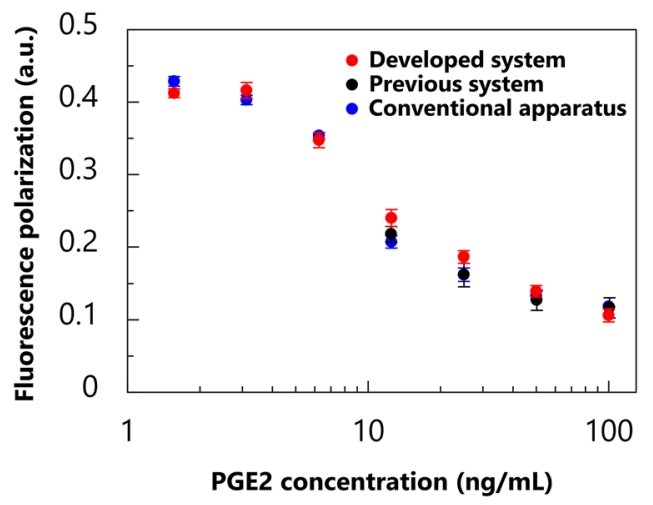

FIG. 3. Standard curve of FP against PGE2 concentration in the range of $1.56-100 \mathrm{ng} / \mathrm{ml}$ obtained from the developed system, previous system, and conventional apparatus.
4 at a time. The measurements were performed in triplicates. Figure 2 shows a typical $P$ image obtained by the FP imaging instruments. The standard curve is obtained from the luminescence of microchannels in such $P$ images in Fig. 3. For comparing performance, the standard curves obtained from our previous system and conventional instrument are also shown in Fig. 3. The coefficient of variations (CVs) from these results is shown in Table I. The reduction of $P$ values was observed with increasing PGE2 concentrations as expected. Additionally, these sets of results properly correlated with each other, confirming FP imaging is possible with the developed system. The error bars of results with the developed system are smaller than that with previous system because of increased LC layer transmittance and improved synchronization. While the maximum standard deviation against $P$ deviation of the conventional FP apparatus is $3.0 \%$, those of developed and previous systems are $3.9 \%$ and $16.1 \%$, respectively. Therefore, the measurement precision of the developed system is almost the same as that of the conventional FP apparatus. By altering the microdevice design,

TABLE I. Percentages of coefficient of variations (CVs) from standard curve in Fig. 3. $\left({ }^{*} 1\right)$ The results cannot to be obtained because of lack of fluorescence intensities.

\begin{tabular}{lccc}
\hline \hline $\begin{array}{l}\text { PGE2 } \\
(\mathrm{ng} / \mathrm{ml})\end{array}$ & $\begin{array}{c}\text { Developed } \\
\text { system }(\%)\end{array}$ & $\begin{array}{c}\text { Previous } \\
\text { system }(\%)^{15}\end{array}$ & $\begin{array}{c}\text { Conventional } \\
\text { apparatus }(\%)\end{array}$ \\
\hline 100 & 7.73 & 10.36 & 0.49 \\
50 & 5.32 & 9.45 & 4.16 \\
25 & 4.30 & 9.01 & 5.72 \\
12.5 & 4.72 & 4.61 & 4.13 \\
6.25 & 2.93 & $* 1$ & 1.07 \\
3.125 & 2.57 & $* 1$ & 1.63 \\
1.5625 & 1.43 & $* 1$ & 1.55 \\
\hline \hline
\end{tabular}


TABLE II. Comparative table of the abilities of the developed system, previous system, and conventional apparatus.

\begin{tabular}{lccc}
\hline \hline & $\begin{array}{c}\text { Developed } \\
\text { system }\end{array}$ & $\begin{array}{c}\text { Previous } \\
\text { system }^{15}\end{array}$ & $\begin{array}{c}\text { Conventional } \\
\text { apparatus }\end{array}$ \\
\hline Number of samples & Multiple & Multiple & Single \\
Precision (\%) & 3.9 & 16.1 & 3.0 \\
Cost & $\sim \$ 13000$ & $\sim \$ 57000$ & $\sim \$ 10000$ \\
\hline \hline
\end{tabular}

it will be easily possible to increase the number of the samples detected simultaneously. The increased throughput is an attractive feature for the molecular interaction analysis field.

\section{CONCLUSIONS}

We developed a new, improved FP imaging system with simultaneous sample processing ability. The simultaneous FPIA of multiple samples for PGE2 was performed to evaluate the measurement performance of the system. From the results, we confirmed that the measurement performance of the developed system is comparable with that of the conventional FP apparatus, improving from the previous system. Table II summarizes and compares the abilities of the three apparatuses (new FP system, previously developed FP system, and the conventional FP system), in terms of the number of samples processed at the same time, measurement precision, and cost. Our developed system is able to carry out simultaneous FP measurement of multiple samples, while the measurement precision of the system is comparable with that of the conventional FP apparatus designed for single analysis. Additionally, we succeeded in cost reduction by using an inexpensive CCD and an LED. Theoretically, our system has potential for further price reduction by optimizing the optical components (lenses and filters), optical arrangement, and electronics used. Moreover, an increase in the number of samples being processed at the same time is possible by changing the microdevice design. We have developed an inexpensive high throughput FP measurement system and hope for its wide usage in molecular interaction studies.

\section{ACKNOWLEDGMENTS}

This work was supported by the JST-SENTAN program. O.W. acknowledges the Grant-in-Aid for JSPS Fellows No. $16 \mathrm{~J} 01317$.

${ }^{1}$ S. Boccaletti, V. Latora, Y. Moreno, M. Chavez, and D.-U. Hwang, Phys. Rep. 424, 175 (2006).

${ }^{2}$ H. Kitano, Science 295, 1662 (2002).

${ }^{3}$ J. Lippincott-Schwartz, E. Snapp, and A. Kenworthy, Nat. Rev. Mol. Cell. Biol. 2, 444 (2001).

${ }^{4}$ M. Cohen, Y. B. Tzur, E. Neufeld, N. Feinstein, M. R. Delannoy, K. L. Wilson, and Y. Gruenbaum, J. Struct. Biol. 140, 232 (2002).

${ }^{5}$ E. A. Jares-Erijman and T. M. Jovin, Nat. Biotechnol. 21, 1387 (2003).

${ }^{6}$ J. R. Lundblad, M. Laurance, and R. H. Goodman, Mol. Endocrinol. 10, 607 (1996).

${ }^{7}$ K. Kakehi, Y. Oda, and M. Kinoshita, Anal. Biochem. 297, 111 (2001).

${ }^{8}$ D. M. Jameson and S. E. Seifried, Methods 19, 222 (1999).

${ }^{9}$ T. Tachi, N. Kaji, M. Tokeshi, and Y. Baba, Lab Chip 9, 966 (2009).

${ }^{10}$ T. Tachi, T. Hase, Y. Okamoto, N. Kaji, T. Arima, H. Matsumoto, M. Kondo, M. Tokeshi, Y. Hasegawa, and Y. Baba, Anal. Bioanal. Chem. 401, 2301 (2011).

${ }^{11}$ Q. Wan and X. C. Le, Anal. Chem. 72, 5583 (2000).

${ }^{12}$ J. Choi, D. Kang, H. Park, A. J. deMello, and S. Chang, Anal. Chem. 84, 3849 (2012).

${ }^{13}$ J. C. Owicki, J. Biomol. Screening 5, 297 (2000).

${ }^{14}$ J. A. Cruz-Aguado and G. Penner, Anal. Chem. 80, 8853 (2008).

${ }^{15}$ O. Wakao, Y. Fujii, M. Maeki, A. Ishida, H. Tani, A. Hibara, and M. Tokeshi, Anal. Chem. 87, 9647 (2015).

${ }^{16}$ M. Maeki, T. Saito, Y. Sato, T. Yasui, N. Kaji, A. Ishida, H. Tani, Y. Baba, H. Harashima, and M. Tokeshi, RSC Adv. 5, 46181 (2015).

${ }^{17}$ N. V. Beloglazova and S. A. Eremin, Talanta 142, 170 (2015).

${ }^{18}$ J. Choi, G. Kim, S. Lee, J. Kim, A. J. deMello, and S. Chang, Biosens. Bioelectron. 67, 497 (2015).

${ }^{19}$ D. M. Aronoff, C. Canetti, and M. Peters-Golden, J. Immunol. 173, 559 (2004).

${ }^{20}$ O. Hayashi, J. Biol. Chem. 263, 14593 (1988). 